\section{FRI0101 EFFECTS OF IL6 INHIBITORS ON THE INCIDENCE OF MAJOR ADVERSE CARDIOVASCULAR EVENTS IN RHEUMATOID ARTHRITIS PATIENTS: A SYSTEMATIC REVIEW WITH META ANALYSIS}

C. Marais ${ }^{1}$, C. Hua ${ }^{1,2}$, E. Filhol ${ }^{1}$, F. Flaisler ${ }^{1}$, C. Lukas ${ }^{2,3}$, J. Morel ${ }^{3}$, C. GaujouxViala ${ }^{1,2} .{ }^{1}$ Department of Rheumatology, Nîmes University Hospital, Nimes, France: ${ }^{2} I D E S P$, University of Montpellier, Montpellier, France; ${ }^{3}$ Department of Rheumatology, CHU Montpellier, University of Montpellier, Montpellier, France

Background: Rheumatoid arthritis (RA) is associated with a 2 fold increased risk of cardiovascular events (CVE) and mortality when compared to the general population. The systemic inflammation in RA seems to play a pivotal role by creating endothelial dysfunction and thus accelerating atherosclerosis. This long lasting inflammatory process potentiates the effects of additional classical cardiovascular risk factors. Since the 2000s, numerous therapeutic advances, in particular biologics, allow better control of this inflammation. Among these, IL6 inhibitors (IL6i) are known to provide rapid and sustained improvements in clinical, biological and radiographic outcomes. However, an increase in circulating lipid concentrations in patients treated with IL6i is usual. This raises the question of the risk -to -benefit ratio of IL6i.

Objectives: The purpose of this systematic literature review and meta-analysis was to evaluate the impact of IL6i on the incidence of major adverse cardiovascular events in RA patients in comparison with TNFalpha inhibitors (TNFi), non TNFi bDMARDs or csDMARDS.

Methods: A systematic literature search of MEDLINE (via PubMed), EMBASE and the Cochrane Library databases until February 2019 was performed. Included studies were observational studies or randomized controlled trials having reported relevant confirmed CVEs (death from CVE, myocardial infarction, heart failure and stroke) in patients with RA treated with IL6i, and a suitable control group. A meta-analysis of the relative risk for each CVE in RA patients treated with IL6i compared to patients in the control groups was performed. A random effect model was applied in case of substantial heterogeneity.

Results: Of 6869 studies, 23 randomized controlled trials and 6 controlled cohorts could be included. IL6i were significantly associated with a reduction in the risk of myocardial infarction in comparison with TNFi (OR, 0.73; 95\% Cl $[0.56$ to 0.96$])$. No other significant effects were observed with regard to the risks of stroke, heart failure (HF), and death from CVE in comparison with csDMARDs, TNFi, or non-TNFi bDMARDs (table 1).

Table 1. Pooled relative risks of cardiovascular events in RA patients treated with IL-6 inhibitors and respective control groups

\begin{tabular}{lccc}
\hline & Cs DMARDS & TNFi & Non TNFi bDMARDS \\
\hline Myocardial infarction & $1.44[0.50 ; 4.17]$ & $0.73[0.56 ; 0.96]$ & $0.81[0.48 ; 1.36]$ \\
Stroke & $1.08[0.40 ; 2.91]$ & $1.20[0.82 ; 1.77]$ & $0.73[0.39 ; 1.37]$ \\
Heart failure & $0.17[0.01 ; 4.08]$ & $1.51[0.61 ; 3.70]$ & $1.19[0.71 ; 1.98]$ \\
Cardiovascular death & $1.59[0.62 ; 4.11]$ & $1.13[0.72 ; 1.78]$ & NA \\
\hline
\end{tabular}

Our findings of a potentially protective effect of IL6i use on the risk of $\mathrm{MI}$ are reassuring. Although several beneficial effects might be involved, like the effective control of systemic inflammation, the anti-arrhythmia effect or the improvement of endothelial and left ventricle dysfunction, a potential indication bias with a decreased likelihood to prescribe these drugs in patients with high cardiovascular risk cannot be excluded.

Conclusion: This review of the literature with meta-analysis provides reassuring results about the association between use of IL6i and CVE in RA patients. Data from long-term observational studies is however needful to confirm and ascertain this result.

Disclosure of Interests: None declared

DOI: 10.1136/annrheumdis-2020-eular.4327

\section{FRI0102 \\ SERUM TNFA LEVELS AT 24 HOURS AFTER FIRST ADMINISTRATION OF CERTOLIZUMAB PEGOL PREDICT EFFECTIVENESS AT WEEK 12 IN PATIENTS WITH RHEUMATOID ARTHRITIS FROM TSUBAME STUDY (UMIN ID:0002381)}

Y. Miyazaki ${ }^{1}$, K. Nakano ${ }^{1}$, S. Nakayamada ${ }^{1}$, S. Kubo ${ }^{1}$, S. Iwata ${ }^{1}$, K. Hanami ${ }^{1}$, S. Fukuyo ${ }^{1}$, I. Miyagawa ${ }^{1}$, A. Yamaguchi ${ }^{1}$, A. Kawabe ${ }^{1}$, S. Kazuyoshi ${ }^{1,2}$, Y. Tanaka ${ }^{1} .{ }^{1}$ School of Medicine, University of Occupational \& Environmental Health, Japan, The First Department of Internal Medicine, Kitakyushu, Japan: ${ }^{2}$ Internal Medicine, Tobata General Hospital, Kitakyushu, Japan

Background: To increase the remission rate of rheumatoid arthritis $(R A)$, it is necessary to determine the efficacy of the tumor necrosis factor (TNF) inhibitor as early as possible. Moreover, the response to certolizumab pegol (CZP) at 12 weeks has been reported to predict its long-term efficacy.
Objectives: As part of a prospective single-center observational study (TSUBAME study), we prospectively enrolled patients to be treated with CZP in our institution to evaluate its effectiveness and safety starting at 24 hours after the first dose in clinical settings, while recording blood CZP concentrations and biomarkers over time to examine their correlation with clinical effects.

Methods: One hundred patients with RA and inadequate response to MTX who received CZP were enrolled in the TSUBAME study. The changes in serum TNFa, IL-6, and CZP levels at 24 hours after first administration of CZP were measured, and the correlation between serum biomarkers and clinical response was determined.

Results: At 24 hours after CZP initiation, significant improvement was observed in the disease activity (baseline and $24 \mathrm{~h}: 5.4 \pm 1.3,5.0 \pm 1.3$ respectively, $p<0.01$ ), which was maintained until week 12 . (baseline and 12 w: $5.4 \pm 1.3,3.3 \pm 1.4$, respectively, $p<0.01$ ). Serum TNFa and IL-6 levels significantly decreased at 24 hours after first administration of CZP compared to baseline. No correlation was found between TNFa and IL-6 levels at baseline and the clinical response. According to univariate analysis, low serum TNFa and IL-6 levels and high CZP levels at 24 hours were associated with DAS28 (ESR) remission at 12 weeks. According to multivariate analysis, low serum TNFa levels at 24 hours were significantly associated with DAS28 (ESR) remission at 12 weeks (OR $0.05,95 \% \mathrm{Cl} 0.01,0.75, \mathrm{p}=$ 0.03 ). Based on these findings, an ROC curve was created using remission according to the DAS28 (ESR) at week 12 as a dependent variable and TNFo concentration at 24 hours as an independent variable, resulting in a cut-of value of $0.76 \mathrm{pg} / \mathrm{ml}$. From this result, the TNFa concentration at 24 hours was divided into 2 groups according to this cut-off, and the rates of remission according to the DAS28 (ESR) at week 12 were compared. In the group with TNFa concentration at 24 hours below the cut-off value, the rate of remission according to the DAS28 (ESR) at week 12 was significantly higher than in the group with TNFa concentration at 24 hours above the cut-off value (below the cut-off: above the cut-off $=56.3 \%: 21.6 \%, p<0.001$ ). Between the group that achieved remission according to the DAS28(ESR) and the group that did not achieve remission at week 12, there was almost no difference in the distribution of TNFa concentrations at baseline; however, the distribution of TNFa concentrations at 24 hours was lower in the group that achieved remission.

Conclusion: CZP was effective where serum TNFa was strongly neutralized within 24 hours. These results suggest that low serum TNFa levels at 24 hours after first administration of CZP may predict the effectiveness of CZP. To increase the remission rate in RA, it is necessary to determine the effectiveness of the molecular targeted drugs used at an early point, in addition to how rapid the onset of action is. CZP is extremely fast-acting, and its effectiveness can be predicted as early as 24 hours after the first dose, suggesting that it may be possible to determine the effectiveness early.

Acknowledgments: The authors thank Ms. M. Hirahara for providing excellent technical assistance.

Disclosure of Interests: Yusuke Miyazaki Grant/research support from: Astellas Pharma Inc and UCB S.A., Kazuhisa Nakano: None declared, Shingo Nakayamada Grant/research support from: Mitsubishi-Tanabe, Takeda, Novartis and MSD, Speakers bureau: Bristol-Myers, Sanofi, Abbvie, Eisai, Eli Lilly, Chugai, Asahi-kasei and Pfizer, Satoshi Kubo: None declared, Shigeru Iwata: None declared, Kentaro Hanami: None declared, Shunsuke Fukuyo: None declared, Ippei Miyagawa: None declared, Ayako Yamaguchi: None declared, Akio Kawabe: None declared, SAITO KAZUYOSHI: None declared, Yoshiya Tanaka Grant/research support from: Asahi-kasei, Astellas, Mitsubishi-Tanabe, Chugai, Takeda, Sanofi, Bristol-Myers, UCB, Daiichi-Sankyo, Eisai, Pfizer, and Ono, Consultant of: Abbvie, Astellas, Bristol-Myers Squibb, Eli Lilly, Pfizer Speakers bureau: Daiichi-Sankyo, Astellas, Chugai, Eli Lilly, Pfizer, AbbVie, YL Biologics, Bristol-Myers, Takeda, Mitsubishi-Tanabe, Novartis, Eisai, Janssen, Sanofi, UCB, and Teijin

DOI: 10.1136/annrheumdis-2020-eular.2252

\section{FRI0103 \\ ASSOCIATION BETWEEN SEROPOSITIVITY AND DISCONTINUATION OF INFLIXIMAB IN RHEUMATOID ARTHRITIS}

Y. Ogawa ${ }^{1}$, N. Takahashi' ${ }^{2}$ T. Kojima ${ }^{2}$, N. Ishiguro ${ }^{2} .{ }^{1}$ Nakatsugawa Municipal General Hospital, Nakatsugawa, Japan; ${ }^{2}$ Nagoya University Graduate School of Medicine, Nagoya, Japan

Background: Infliximab is still a widely used biologic agent in treatment of rheumatoid arthritis (RA). Because infliximab is expensive and can have adverse events, identification of factors that predict an adequate response to this treatment has been investigated.

Objectives: In this study, we investigated the association between rheumatoid factor (RF) and anti-citrullinated protein antibodies (ACPA) status and 